\title{
Real-Time Offset Error Compensation of 6D IMU Mounted on Ground Vehicles Using Disturbance Observer
}

\author{
Jiwon Oh, Yoonkab Noh, and Seibum B. Choi
}

\begin{abstract}
This paper mainly deals with the offset error compensation algorithm related with the 6D IMU (inertial measurement unit) that measures the linear accelerations and angular velocities about the longitudinal, lateral, and vertical axis of ground vehicles. It is assumed that the independent wheel speed data and steering wheel angle information are provided for the sensor compensation algorithm. Using a disturbance observer, through designing a linear model and inverse model of the vehicle motion, the offset errors of the accelerometers are estimated. The stability of the entire compensational system is verified, and finally, the performance of the suggested algorithm is tested based on a well-known vehicle dynamics simulation tool, CarSim.
\end{abstract}

Index Terms-Bicycle model, disturbance observer, inertial measurement unit, offset compensation, signal processing.

\section{INTRODUCTION}

The advancement of computerized technology is encouraging the production of the vehicles with electronic safety systems mounted. To guarantee proper operation of these systems such as the localization systems [1]-[5], and the vehicle dynamics aiding systems that may include ESP (electronic stability program) and ROM (roll over mitigation), it is crucial that the sensor measurement information is reliable enough. Unfortunately, however, it must be noted that the highly accurate sensors are only available at a high cost.

Thus, to break this tradeoff between cost and performance of the sensors, this paper presents a novel method to minimize the sensor offset error. Knowing that the offset error is the biggest issue concerning sensor signal integration, and that estimation of the vehicle states - perhaps side slip angle, roll, and pitch angle - needed for proper electronic safety system operation requires numerical integration of the sensor signals, elimination of the offset error can significantly increase the accuracy of the state estimation.

The main contribution that distinguishes this paper from others is the accurate dynamic estimation of the sensor offset errors, which does not require attitude initialization or GPS signals. Also, another contribution is the offset error estimation robustness in the influence of severe vehicle motion (side slip angle). Such performance is obtained through the use of the lateral velocity estimation from the vehicle model-based observer which utilizes the compensated lateral acceleration measurement, and the stability of this coupled dynamics is analyzed to guarantee accurate sensor

Manuscript received November 9, 2012; revised January 24, 2013.

The authors are with Korea Advanced Institute of Science and Technology, Kuseong, Yuseong, Daejeon, Korea (tel.: +82-42-350-4160; fax: +82-42-350-4110; e-mail: jwo@kaist.ac.kr). offset error identification without steady state error. This unique attempt has not been used in the previous works of the similar interest [6]-[11] that require additional sensor information or GPS corrections.

The basic organization of this paper is as follows. Section II A describes the gyroscope offset compensation which is a prerequisite for the accelerometer offset error compensation procedure. Section II B introduces a bicycle model-based observer of a vehicle as the linear model of the sensor signal compensation system. Section II C then introduces the inverse model that is coupled with the previously mentioned linear model of the vehicle as parts of the disturbance observer. Section III provides the stability analysis of the entire system, and Section IV presents the simulation results based on CarSim to validate the sensor error correction performance.

\section{OBSERVER DESIGN}

\section{A. Gyroscope Offset Compensation}

The gyroscope offset error compensation is much simpler than the accelerometer offset compensation, since the angular velocities of the vehicle at stop are zero regardless of the vehicle attitude, and the long-term average of the vehicle roll and pitch angles can be assumed to be zero. Using these properties, the roll and pitch rate sensors can be easily adjusted as shown in the following for the sensor errors $e_{p}$ and $e_{q}$.

$$
\begin{gathered}
p(t)=p^{*}(t)+e_{p}(t) \\
\dot{e}_{p}=\gamma_{p}\left[-e_{p}-p^{*}(t)\right] \\
q(t)=q^{*}(t)+e_{q}(t) \\
\dot{e}_{q}=\gamma_{q}\left[-e_{q}-q^{*}(t)\right]
\end{gathered}
$$

Here, $p^{*}(t)$ and $q^{*}(t)$ are the raw roll and pitch rate signals, whereas $p(t)$ and $q(t)$ are the processed ones. $\gamma_{p}$ and $\gamma_{q}$ are the positive tuning constants.

Because the yaw rate may be a steady state non-zero value, the correctional scheme for yaw rate is only applied by referring to the steering wheel angle, $\delta_{f}$.

$$
\begin{gathered}
r(t)=r^{*}(t)+e_{r} \\
\dot{e}_{r}=Z_{r}(t) \gamma_{r}\left[-e_{r}-r^{*}(t)\right]
\end{gathered}
$$


where $Z_{r}(t)=\left\{\begin{array}{l}1,\left|\delta_{f}\right| \leq k_{r} \\ 0,\left|\delta_{f}\right|>k_{r}\end{array}\right.$

In the above compensation scheme, $r^{*}(t)$ is the measured raw yaw rate, whereas $r(t)$ is the processed one. $\gamma_{r}$ and $k_{r}$ are the positive tuning constants, and $e_{r}$ is the estimated yaw rate sensor error.

A significant benefit of dynamically zeroing the gyroscope sensors is that, through applying the suggested algorithm with the time window, the compensation algorithm automatically adjusts the measurements even in cases of time-varying offset errors.

\section{B. Bicycle Model-Based Observer}

To model the lateral dynamics of the vehicle, the following linear bicycle model is considered.

$$
\dot{x}=A x+B u
$$

where $x=\left[\begin{array}{c}\beta \\ r\end{array}\right], u=\delta_{f}$,

$A=\left[\begin{array}{ll}a_{11} & a_{12} \\ a_{21} & a_{22}\end{array}\right]=\left[\begin{array}{cc}-\frac{2\left(C_{f}+C_{r}\right)}{m v_{x}} & \frac{-2\left(C_{f} l_{f}-C_{r} l_{r}\right)}{m v_{x}^{2}}-1 \\ \frac{-2\left(C_{f} l_{f}-C_{r} l_{r}\right)}{I_{z}} & -\frac{2\left(C_{f} l_{f}^{2}+C_{r} l_{r}^{2}\right)}{I_{z} v_{x}}\end{array}\right]$,

and $B=\left[\begin{array}{l}b_{1} \\ b_{2}\end{array}\right]=\left[\begin{array}{c}\frac{2 C_{f}}{m v_{x}} \\ \frac{2 C_{f} l_{f}}{I_{z}}\end{array}\right]$

Concerning the implementation of the bicycle model shown in (1), the cornering stiffness $C_{f}$ and $C_{r}$ henceforth denote those obtained by the cornering stiffness adaptation scheme dealt in [12].

The lateral acceleration is expressed as the following.

$$
a_{y}=\dot{v}_{y}+r v_{x}
$$

Through substituting the right hand side of (8) with the terms used in (7), the lateral acceleration can finally be expressed as shown in (9) [13].

$$
a_{y}=a_{11} v_{x} \beta+\left(a_{12}+1\right) v_{x} r+b_{1} v_{x} \delta_{f}
$$

Here, the influence of vehicle suspension angle and road bank angle on the lateral acceleration measurement is considered [14]. Besides, the possible influence of the vehicle vertical motion is not considered in the bicycle model observer, since its contribution is assumed to be negligible.

Making use of (8) and choosing the yaw rate and the lateral acceleration as the system outputs, the following estimated output equations can be set up.

$$
\begin{aligned}
& \hat{y}=C \hat{x}+D u \\
& \text { where } \quad \hat{y} \triangleq\left[\begin{array}{c}
\hat{r} \\
\hat{a}_{y}
\end{array}\right], x \triangleq\left[\begin{array}{c}
\hat{\beta}_{b i c} \\
\hat{r}
\end{array}\right], \\
& C=\left[\begin{array}{cc}
0 & 1 \\
a_{11} v_{x} & \left(a_{12}+1\right) v_{x}
\end{array}\right], \text { and } D=\left[\begin{array}{c}
0 \\
b_{1} v_{x}
\end{array}\right]
\end{aligned}
$$

With these, and substituting the longitudinal velocity with that estimated based on the wheel dynamics, the following observer is designed.

$$
\begin{aligned}
& \dot{\hat{x}}=A \hat{x}+B u+K(y-\hat{y}) \\
& K \triangleq\left[\begin{array}{ll}
K_{1} & K_{2} \\
K_{3} & K_{4}
\end{array}\right]
\end{aligned}
$$

Expanding (11) gives the following:

$$
\begin{gathered}
{\left[\begin{array}{c}
\dot{\hat{\beta}}_{b i c} \\
\dot{\hat{r}}
\end{array}\right]=\left[\begin{array}{ll}
a_{11}-K_{2} a_{11} v_{c a r} & a_{12}-K_{1}-K_{2}\left(a_{12}+1\right) v_{c a r} \\
a_{21}-K_{4} a_{11} v_{c a r} & a_{22}-K_{3}-K_{4}\left(a_{12}+1\right) v_{c a r}
\end{array}\right]\left[\begin{array}{c}
\hat{\beta}_{b i c} \\
\hat{r}
\end{array}\right]} \\
+\left[\begin{array}{l}
b_{1}-K_{2} b_{1} v_{c a r} \\
b_{2}-K_{4} b_{1} v_{c a r}
\end{array}\right] \delta_{f}+\left[\begin{array}{ll}
K_{1} & K_{2} \\
K_{3} & K_{4}
\end{array}\right]\left[\begin{array}{c}
r \\
a_{y}
\end{array}\right]
\end{gathered}
$$

where $\hat{\beta}_{b i c}, \hat{r}, r$ and $a_{y}$ are the estimated side slip angle, estimated yaw rate, sensor yaw rate measurement, and compensated lateral acceleration measurement, respectively. Assuming that the wheel slip is negligible, $v_{x}$ is replaced by $v_{c a r}$, the selected and filtered value obtained based on the four independent wheel speed data.

Now, using the frozen-time pole-placement method, the observer gain $K$ is selected to guarantee the observer system stability.

$$
\left[\begin{array}{cc}
K_{1} & K_{2} \\
K_{3} & K_{4}
\end{array}\right]=\left[\begin{array}{cc}
\frac{I_{z}\left(C_{f} l_{f}-C_{r} l_{r}\right)}{2 C_{f} C_{r}\left(l_{f}+l_{r}\right)^{2}} p_{o}^{2}-1 & \frac{1}{v_{c a r}} \\
-2 p_{o} & \frac{m\left(C_{f} l_{f}^{2}+C_{r} l_{r}^{2}\right)}{I_{z}\left(C_{f} l_{f}-C_{r} l_{r}\right)}
\end{array}\right]
$$

where $p_{o}$ is a negative constant. For the sake of maintaining the system stability, the values of $K_{2}$ and $K_{4}$ are switched to zero as their denominators closely approach zero [14]. The bicycle model observer thus estimates the vehicle lateral velocity using the following relationship.

$$
\hat{v}_{y, b i c}=v_{c a r} \tan \hat{\beta}_{\text {bic }}
$$

where $\hat{v}_{y, b i c}$ is the lateral velocity estimation obtained from the bicycle model-based observer.

\section{Formation of Inverse Kinematic Model and Accelerometer Offset Compensation}

The gyroscope offset correction may be done at the moment the car starts, since the true angular velocities are surely zero when the vehicle is at a complete stop. However, the accelerometer compensation cannot be done this way. The true accelerometer measurement of a vehicle depends on the vehicle attitude, i.e., initial conditions for the acceleration vary and are unknown because they are influenced by gravity. For this reason, the accelerometer error must be compensated dynamically as the vehicle moves.

If the previously explained linear bicycle model-based observer gives the side slip angle and thus the vehicle lateral velocity by using the vehicle acceleration input, the inverse model used for disturbance rejection outputs the vehicle acceleration values when the vehicle velocities are fed. This principle is described using the block diagram shown in Fig. 1. 
Since we need the acceleration compensation of all three axes, the kinematic relationship is used as shown next.

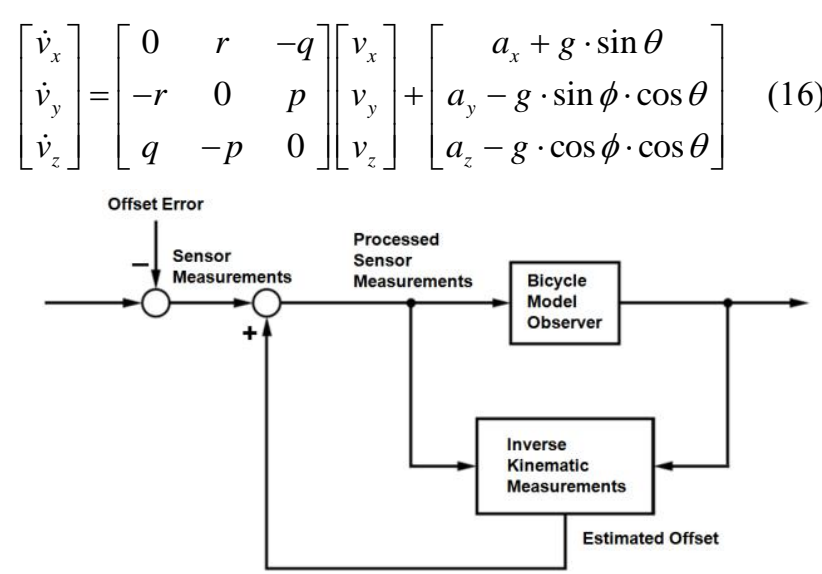

Fig. 1. Block diagram representation of the accelerometer offset error compensation.

Separating the acceleration terms, the following equations are obtained.

$$
\begin{gathered}
a_{x}=\dot{v}_{x}-r \cdot v_{y}+q \cdot v_{z}-g \cdot \sin \theta \\
a_{y}=\dot{v}_{y}+r \cdot v_{x}-p \cdot v_{z}+g \cdot \sin \phi \cdot \cos \theta \\
a_{z}=\dot{v}_{z}-q \cdot v_{x}+p \cdot v_{y}+g \cdot \cos \phi \cdot \cos \theta
\end{gathered}
$$

Here, it is assumed that $v_{x}=v_{c a r}$, and $v_{y}=\hat{v}_{y, b i c}$. Also, $v_{z}=0, \phi=0$, and $\theta=0$, since the long term average of the indicated vehicle states even out to zero. Incorporating the above mentioned assumptions, the reference acceleration values can be defined as follows.

$$
\begin{gathered}
a_{x, r e f} \equiv \dot{v}_{c a r}-r \cdot \hat{v}_{y, r e f} \\
a_{y, r e f} \equiv \dot{\hat{v}}_{y, r e f}+r \cdot v_{c a r} \\
a_{z, r e f} \equiv-q \cdot v_{c a r}+p \cdot \hat{v}_{y, r e f}+g
\end{gathered}
$$

By comparing the above values to the raw acceleration measurements, the raw signals are processed according to the following schemes.

$$
\begin{gathered}
a_{x}(t)=a_{x}^{*}(t)+e_{x}(t) \\
\dot{e}_{x}=Z_{x}(t) \gamma_{x}\left[-e_{x}+\left(a_{x, r e f}-a_{x}^{*}\right)\right]
\end{gathered}
$$

where $Z_{x}(t)=\left\{\begin{array}{l}1, \mathrm{x} \text {-axis dynamic sensor zeroing condition } \\ 0, \text { otherwise }\end{array}\right.$

$$
\begin{gathered}
a_{y}(t)=a_{y}^{*}(t)+e_{y}(t) \\
\dot{e}_{y}=Z_{y}(t) \gamma_{y}\left[-e_{y}+c_{y}\left(a_{y, r e f}-a_{y}^{*}\right)\right]
\end{gathered}
$$

where $Z_{y}(t)=\left\{\begin{array}{l}1, \text { y-axis dynamic sensor zeroing condition } \\ 0, \text { otherwise }\end{array}\right.$

$$
a_{z}(t)=a_{z}^{*}(t)+e_{z}(t)
$$

$$
\dot{e}_{z}=Z_{z}(t) \gamma_{z}\left[-e_{z}+\left(a_{z, r e f}-a_{z}^{*}\right)\right]
$$

where $Z_{z}(t)=\left\{\begin{array}{l}1, \text { z-axis dynamic sensor zeroing condition } \\ 0, \text { otherwise }\end{array}\right.$

Here, $\gamma_{x}, \gamma_{y}, c_{y}$, and $\gamma_{z}$ are the positive tuning constants, with $c_{y}<1$. The dynamic sensor zeroing condition is discriminated to apply the correction algorithm only during the steady state condition.

\section{STABILITY ANALYSIS}

In this section, the stability of the accelerometer offset compensation algorithm is analyzed. Note that the stability analysis of the longitudinal and vertical acceleration offset compensation is trivial, since their reference values do not involve any feedback. However, the lateral accelerometer error compensation surely forms a loop in the disturbance observer, which requires the stability criterion to be met for robust performance.

$$
\begin{array}{r}
{\left[\begin{array}{c}
\dot{\hat{\beta}}_{b i c} \\
\dot{\hat{r}} \\
\dot{e}_{y}
\end{array}\right]=\left[\begin{array}{ccc}
a_{11}-K_{2} a_{11} v_{c a r} & a_{12}-K_{1}-K_{2}\left(a_{12}+1\right) v_{c a r} & K_{2} \\
a_{21}-K_{4} a_{11} v_{c a r} & a_{22}-K_{3}-K_{4}\left(a_{12}+1\right) v_{c a r} & K_{4} \\
0 & 0 & -\gamma_{y}
\end{array}\right]\left[\begin{array}{c}
\hat{\beta}_{b i c} \\
\hat{r} \\
e_{y}
\end{array}\right]} \\
+\left[\begin{array}{c}
b_{1}-K_{2} b_{1} v_{c a r} \\
b_{2}-K_{4} b_{1} v_{c a r} \\
0
\end{array}\right] \delta_{f}+\left[\begin{array}{ccc}
K_{1} & K_{2} & 0 \\
K_{3} & K_{4} & 0 \\
c_{y} \gamma_{y} \cdot v_{c a r} & -c_{y} \gamma_{y} & 1
\end{array}\right]\left[\begin{array}{c}
r \\
a_{y m} \\
c_{y} \gamma_{y} v_{c a r} \dot{\hat{\beta}}_{b i c}
\end{array}\right]
\end{array}
$$

Recall (14) for the stability verification. The error dynamics of the lateral acceleration offset value must be considered along with the bicycle model observer dynamics which estimates the lateral velocity.

Eq. (29) shows the observer system which has merged the bicycle model observer and the lateral accelerometer error dynamics. Rearranging the above relationship gives the following.

$$
\begin{aligned}
& {\left[\begin{array}{c}
\dot{\hat{\beta}}_{b i c} \\
\dot{\hat{r}} \\
\dot{\hat{e}}_{y}
\end{array}\right]=\left\{\begin{array}{ccc}
a_{11}-K_{2} a_{11} v_{c a r} & a_{12}-K_{1} & K_{2} \\
a_{21}-K_{4} a_{11} v_{c a r} & \left.a_{22}-K_{3}+1\right) v_{c a r} & \\
c_{y} \gamma_{y} v_{c a r}\left(\begin{array}{l}
a_{11} \\
-K_{2} a_{11} v_{c a r}
\end{array}\right) & -K_{4}\left(a_{12}+1\right) v_{c a r} & K_{4} \gamma_{y} v_{c a r}\left(\begin{array}{l}
a_{12}-K_{1} \\
-K_{2}\left(a_{12}+1\right) v_{c a r}
\end{array}\right) \\
c_{y} \gamma_{y} v_{c a r} K_{2} \\
-\gamma_{y}
\end{array}\right]} \\
& \left.\cdot\left[\begin{array}{c}
\hat{\beta}_{b i c} \\
\hat{r} \\
\hat{e}_{y}
\end{array}\right]\right\}+\left[\begin{array}{c}
b_{1}-K_{2} b_{1} v_{c a r} \\
b_{2}-K_{4} b_{1} v_{c a r} \\
c_{y} \gamma_{y} v_{c a r} b_{1}-c_{y} \gamma_{y} v_{c a r} K_{2} b_{1} v_{c a r}
\end{array}\right] \delta_{f} \\
& +\left[\begin{array}{cc}
K_{1} & K_{2} \\
K_{3} & K_{4} \\
c_{y} \gamma_{y} v_{c a r} K_{1}+c_{y} \gamma_{y} \cdot v_{c a r} & c_{y} \gamma_{y} v_{c a r} K_{2}-c_{y} \gamma_{y}
\end{array}\right]\left[\begin{array}{c}
r \\
a_{y m}
\end{array}\right]
\end{aligned}
$$

This can be compared to the ideal system shown next.

$$
\left[\begin{array}{c}
\dot{\beta}_{b i c} \\
\dot{r} \\
\dot{e}_{y}
\end{array}\right]=\left[\begin{array}{ccc}
a_{11} & a_{12} & 0 \\
a_{21} & a_{22} & 0 \\
0 & 0 & 0
\end{array}\right]\left[\begin{array}{c}
\beta_{b i c} \\
r \\
e_{y}
\end{array}\right]+\left[\begin{array}{c}
b_{1} \\
b_{2} \\
0
\end{array}\right] \delta_{f}
$$

There is no dynamics involved in $e_{y}$, since it is assumed 
that the ideal $e_{y}$ is slowly varying. Now to compare the observer estimation with the real states, the errors are defined.

$$
\left[\begin{array}{c}
\dot{\tilde{\beta}}_{b i c} \\
\dot{\tilde{r}} \\
\dot{\tilde{e}}_{y}
\end{array}\right] \triangleq\left[\begin{array}{c}
\dot{\beta}_{b i c}-\dot{\hat{\beta}}_{b i c} \\
\dot{r}-\dot{\hat{r}} \\
\dot{e}_{y}-\dot{\hat{e}}_{y}
\end{array}\right]
$$

With the above, the following error dynamics is reached.

$$
\begin{aligned}
& {\left[\begin{array}{c}
\dot{\tilde{\beta}}_{b i c} \\
\dot{\tilde{r}} \\
\dot{\tilde{e}}_{y}
\end{array}\right]=\left[\begin{array}{ccc}
K_{2} a_{11} v_{c a r} & K_{1}+K_{2}\left(a_{12}+1\right) v_{c a r} & -K_{2} \\
K_{4} a_{11} v_{c a r} & K_{3}+K_{4}\left(a_{12}+1\right) v_{c a r} & -K_{4} \\
0 & -c_{y} \gamma_{y} v_{c a r}\left(\begin{array}{c}
a_{12}-K_{1} \\
-\left(a_{12}+1\right)
\end{array}\right) & \gamma_{y}\left(1-c_{y}\right)
\end{array}\right]\left[\begin{array}{c}
\tilde{\beta}_{b i c} \\
\tilde{r} \\
\tilde{e}_{y}
\end{array}\right]} \\
& +\left[\begin{array}{c}
0 \\
K_{4} b_{1} v_{c a r} \\
0
\end{array}\right] \delta_{f}+\left[\begin{array}{cc}
-K_{1} & -K_{2} \\
-c_{y} \gamma_{y} v_{c a r} K_{1}-c_{y} \gamma_{y} \cdot v_{c a r} & 0
\end{array}\right]\left[\begin{array}{c}
r \\
a_{y m}
\end{array}\right]
\end{aligned}
$$

Through an investigation on the state matrix, it turns out that it is strictly Hurwitz. Hence, it shows the stability of the combined system. Mere stability, however, does not guarantee the ideal offset compensation of the sensor measurements. In order to show the asymptotic stability, (33) is further extended to have the steady state analysis obtained.

At a steady state, it is assumed that there is no change in the states, so the following relationship holds.

$$
\begin{aligned}
& {\left[\begin{array}{l}
0 \\
0 \\
0
\end{array}\right]=\left[\begin{array}{ccc}
K_{2} a_{11} v_{c a r} & K_{1}+K_{2}\left(a_{12}+1\right) v_{c a r} & -K_{2} \\
K_{4} a_{11} v_{c a r} & K_{3}+K_{4}\left(a_{12}+1\right) v_{c a r} & -K_{4} \\
0 & -c_{y} \gamma_{y} v_{c a r}\left(\begin{array}{c}
a_{12}-K_{1} \\
-\left(a_{12}+1\right)
\end{array}\right) & \gamma_{y}\left(1-c_{y}\right)
\end{array}\right]\left[\begin{array}{c}
\tilde{\beta}_{b i c} \\
\tilde{r} \\
\tilde{e}_{y}
\end{array}\right]} \\
& +\left[\begin{array}{cc}
-K_{1} & -K_{2} \\
-K_{3} & -K_{4} \\
-c_{y} \gamma_{y} v_{c a r} K_{1}-c_{y} \gamma_{y} \cdot v_{c a r} & 0
\end{array}\right]\left[\begin{array}{c}
r \\
a_{y m}
\end{array}\right]
\end{aligned}
$$

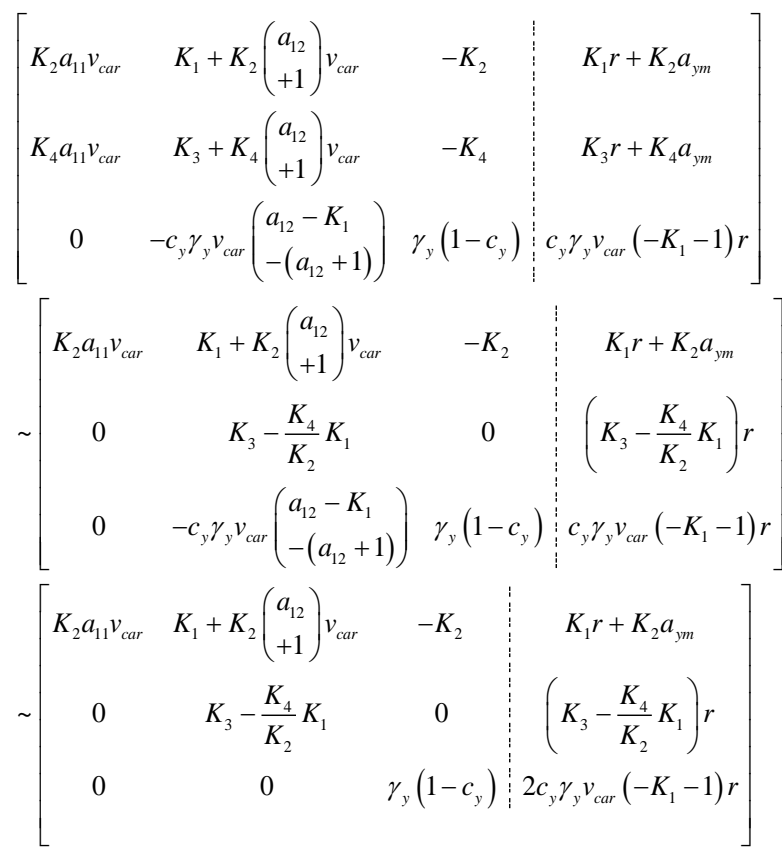

In order to solve for the state errors, the above relationship is put into an augmented matrix and row operations are applied to reach the echelon form.

From the result obtained in (35), it is straight forward to obtain the steady state errors of the states.

$$
\left.\tilde{e}_{y}\right|_{s s}=\frac{2 c_{y} \gamma_{y} v_{c a r}\left(-K_{1}-1\right) r}{\gamma_{y}\left(1-c_{y}\right)}
$$

$$
\left.\tilde{r}\right|_{s s}=r
$$

$$
\left.\tilde{\beta}_{b i c}\right|_{s s}=\frac{K_{1} r+K_{2} a_{y m}+K_{2} \tilde{e}_{y . s s}-\left(K_{1}+K_{2}\left(a_{12}+1\right) v_{c a r}\right) \tilde{r}}{a_{11}}
$$

If the dynamic zeroing condition $Z_{y}(t)$ is set to 1 only when the yaw rate is close to zero, the compensation system satisfies the steady state stability.

\section{Simulation Results}

Using CarSim, the 6D IMU sensor offset error compensation is performed with two scenarios. The first condition deals with accelerating the vehicle and performing four consecutive severe double lane changes. This is to test the accelerometer measurement compensation performance under the influence of severe vehicle motion.

The second scenario deals with starting the vehicle on the inclined land, accelerating the car to $100 \mathrm{~km} / \mathrm{h}$, encountering another severe longitudinally and laterally inclined road, and spinning out of the road. This scenario is to test the compensation performance under the effect of, firstly, initial inclination, and secondly, road bank, hill, and severe lateral motion combined.
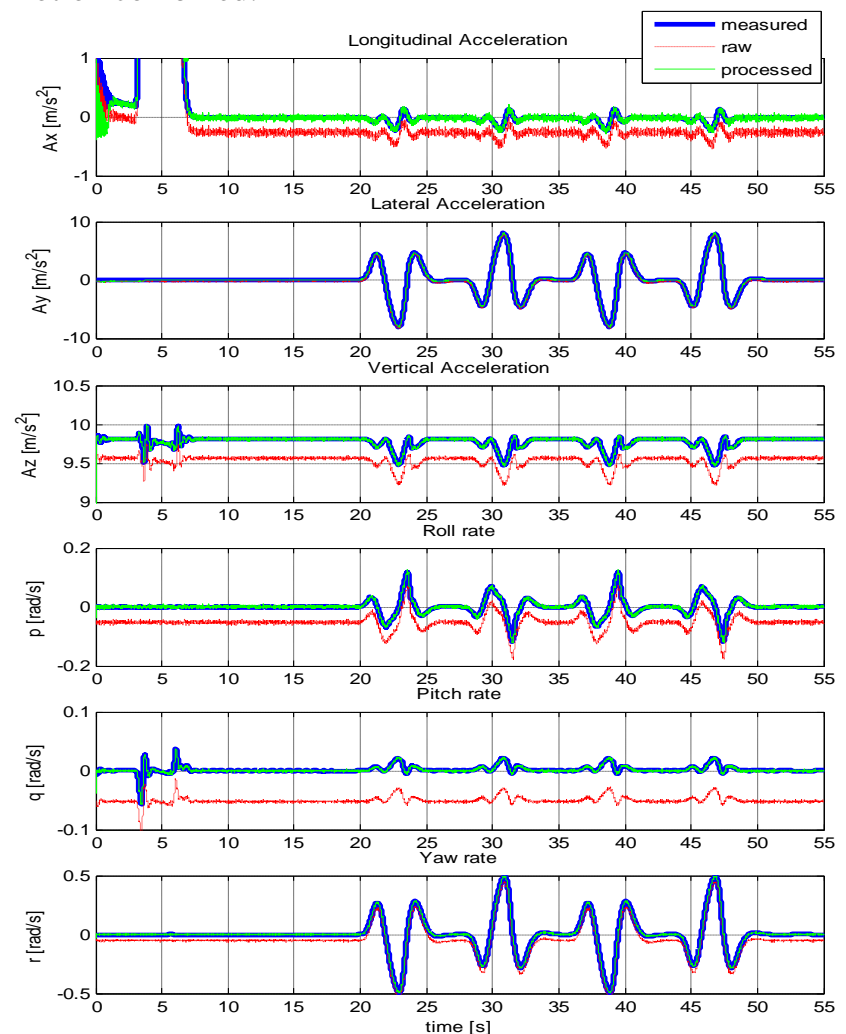

Fig. 2. 6D IMU offset error compensation results: flat road start and four consecutive double lane changes 
For both cases, significant amount of noise and offset error are intentionally added to the ideal sensor measurement values so that a low cost IMU performance can be simulated.

As Fig. 2 shows, the suggested algorithm compensates the raw signals fairly accurately, that the processed result is almost identical to the ideal value. This can be more clearly seen in Fig. 3 where the errors are plotted.
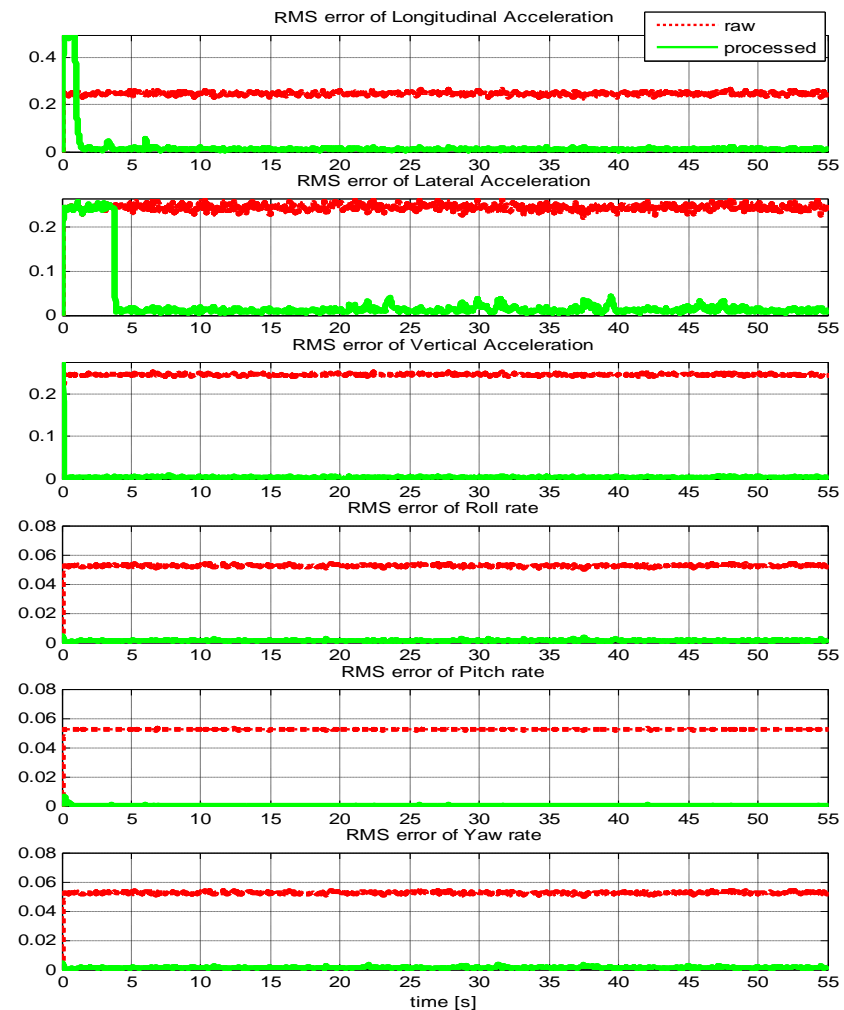

Fig. 3. RMS ( $0.1 \mathrm{~s})$ errors of the raw and processed measurements for the first scenario
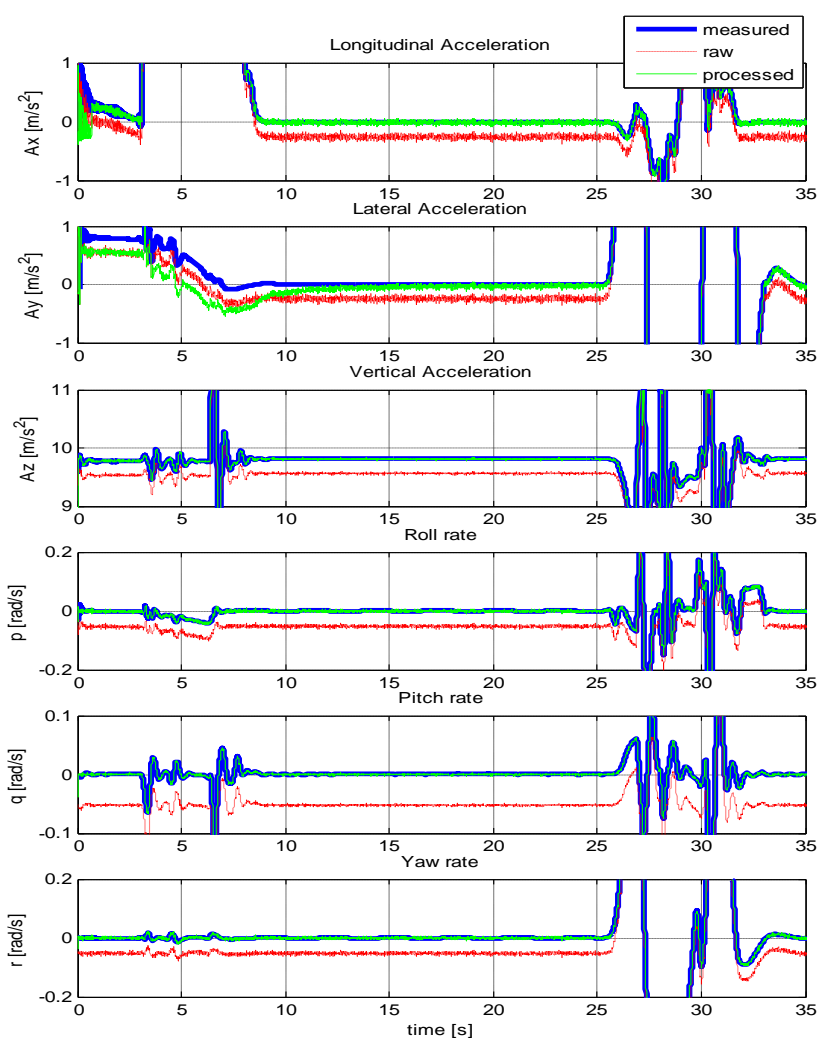

Fig. 4. 6D IMU offset error compensation results: inclined road start and spin out on inclined surface
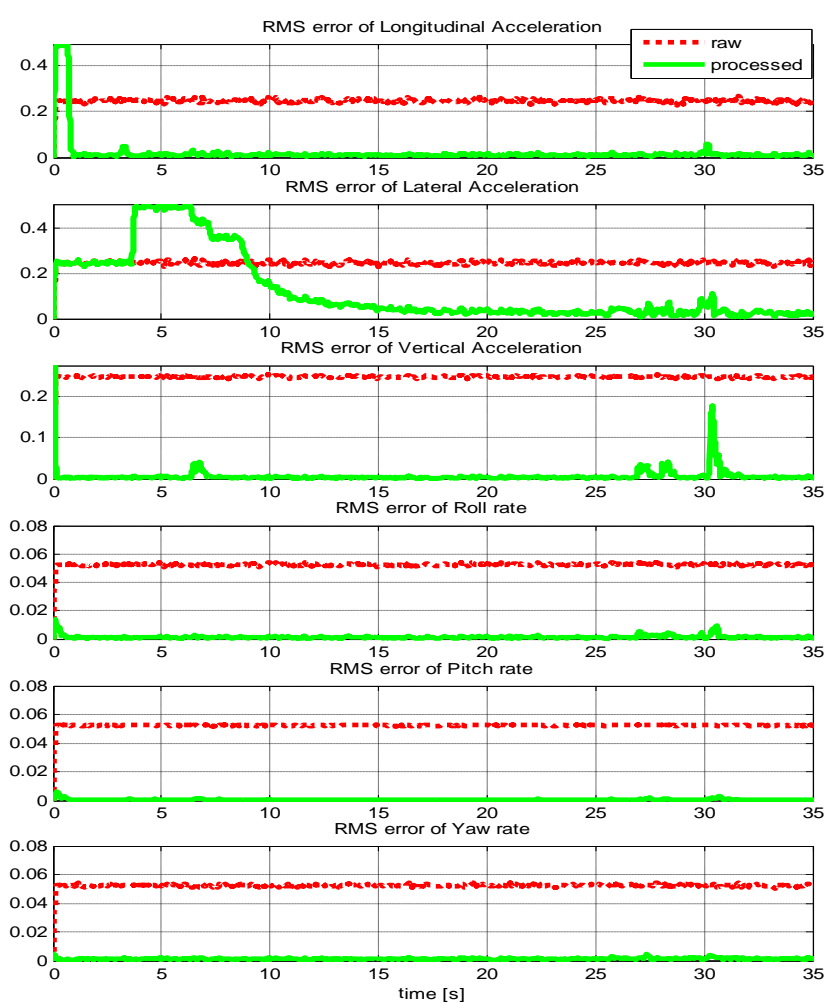

Fig. 5. RMS $(0.1 \mathrm{~s})$ errors of the raw and processed measurements for the second scenario

In case of the second scenario, Fig. 4 shows that the error for the lateral accelerometer compensation momentarily increases as the vehicle starts to move, due to the initial bank angle, but as expected, it is corrected dynamically as the vehicle engages in motion. Once this dynamic sensor zeroing is done, it can be seen in fig. 5 that the errors stay low even under the additional influence of the inclined attitude or severe side slip starting from about 25 second.

\section{CONCLUSION}

This paper presents a novel real-time sensor offset error compensation scheme based on the principle of disturbance observer. Without having to know the initial condition, the gyroscope as well as accelerometer offset error are estimated and corrected as the vehicle engages in motion, only by using the raw 6D IMU measurements and basic vehicle information. This method is tested under various conditions using CarSim, and the algorithm is proven to be useful even in the existence of road angle and severe steering input.

\section{REFERENCES}

[1] S. B. Kim, K. H. Choi, S. Y. Lee, J. H. Choi, T. H. Hwang, B. T. Jang, and J. H. Lee, "A bimodal approach for land vehicle localization," ETRI Journal, vol. 26, no. 5, pp. 497-500, Oct. 2004.

[2] Y. Li, J. Wang, C. Rizos, G. Baitch, and D. Kinlyside, "Application of GPS/INS integration technique to bushfire monitoring," in Proc. Spatial Sciences Conference, Melbourne, Australia, 12-16, Sept. 2005, pp. 1394-1403.

[3] S. Y. Cho and W. S. Choi, "A robust positioning technique in DR/GPS using the receding horizon sigma point kalman FIR filter," in Proc. the IEEE IMTC 2005, pp. 1354-1359, 2005.

[4] S. Y. Cho and W. S. Choi, "Performance enhancement of low-cost land navigation for location based service," ETRI Journal, vol. 28, no. 2, pp. 131-144, Apr. 2006.

[5] A. Noureldin, B. Karamat, M. D. Eberts, and A. El-Shafie, "Performance enhancement of MEMS-based INS/GPS integration for 
low-cost navigation applications," IEEE Transactions on Vehicular Technology, vol. 58, no. 3, 2009.

[6] H. Tanaka and Y. Kazumi, "Gravitational accelerometer provided with zero adjuster," U.S. patent 5526263, 1996.

[7] R. M. Rogers, "IMU in-motion alignment without benefit of attitude initialization," Navigation: Journal of the Institute of Navigation, vol. 44, no. 3, pp. 301-311, 1997

[8] J. D. Begin and K. C. Cheok, "Calibration of multi-axis accelerometer in vehicle navigation system," U.S. patent 6532419, 1998.

[9] E. H. Shin, "Accuracy improvement of low cost INS/GPS for land applications," UCGE Reports Number 20156, The University of Calgary, Calgary, Alberta, Canada, Dec 2001.

[10] E. H. Shin and N. El-Sheimy, "An unscented kalman filter for in-motion alignment of low-cost IMUs," in Proc. IEEE Frames Conference, pp. 273-279, 2004.

[11] W. Abdel-Hamid, T. Abdelazim, N. El-Sheimy, and G. Lachapelle, "Improvement of MEMS-IMU/GPS performance using fuzzy modeling," GPS Solution, vol. 10, no. 2, pp. 1-11, 2006.

[12] S. H. You, J. O. Hahn, and H. C. Lee, "New adaptive approaches to real-time estimation of vehicle sideslip angle," Control Engineering Practice, vol. 17, no. 12, 1367-1379, 2009.

[13] H. W. Son, "A study on design of a robust vehicle side-slip angle observer using an integration of kinematic and bicycle model," in Proc. the KSAE 2008 Annual Conference, Daejeon, Korea, 2008.

[14] J. Oh and S. B. Choi, "Design of a new composite observer for vehicle velocity and attitude estimation," in Proc. the IASTED International Conference, control and Applications, Vancouver, BC, Canada, 2011, pp. 102-109.

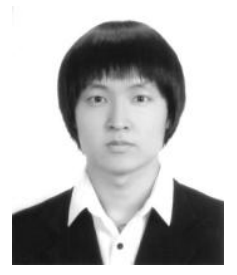

Jiwon Oh obtained his Bachelor's degree in mechanical engineering from Korea Advanced Institute of Science and Technology (KAIST), Daejeon, Korea. He obtained his M.S. degree from the same institution in 2012. He is currently a Ph.D. course student in automotive control laboratory of mechanical engineering department at KAIST. His research interests include vehicle state estimation and control.

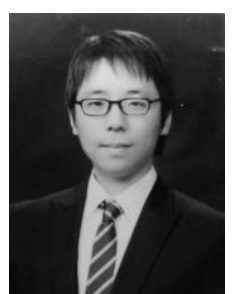

Yoonkab Noh obtained his Bachelor's degree in electrical engineering from Sungkyunkwan University, Suwon, Korea. He is currently a M.S. course student in automotive control laboratory of mechanical engineering department at KAIST. His research interests include vehicle state estimation and control.

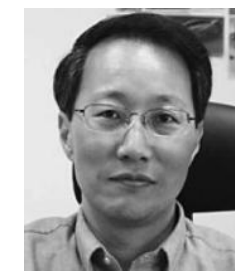

Seibum B. Choi (M'09) received the B.S. degree in mechanical engineering from Seoul National University, Seoul, Korea, the M.S. degree in mechanical engineering from Korea Advanced Institute of Science and Technology (KAIST), Daejeon, Korea, and the Ph.D. degree in controls from the University of California, Berkeley, in 1993. From 1993 to 1997, he was involved in the development of automated vehicle control systems at the Institute of transportation Studies, University of California, Berkeley. During 2006, he was with TRW, Warren, MI, where he was engaged in the development of advanced vehicle control systems. Since 2006, he has been with the faculty of the Mechanical Engineering Department, KAIST. His current research interests include fuel saving technology, vehicle dynamics and control, and active safety systems. 